\title{
On the classification problem for split graphs
}

\author{
Sheila Morais de Almeida • Célia Picinin de Mello • \\ Aurora Morgana
}

Received: 5 October 2011 / Accepted: 20 October 2011 / Published online: 9 November 2011

(C) The Brazilian Computer Society 2011

\begin{abstract}
The Classification Problem is the problem of deciding whether a simple graph has chromatic index equal to $\Delta$ or $\Delta+1$. In the first case, the graphs are called Class 1, otherwise, they are Class 2. A split graph is a graph whose vertex set admits a partition into a stable set and a clique. Split graphs are a subclass of chordal graphs. Figueiredo at al. (J. Combin. Math. Combin. Comput. 32:79-91, 2000) state that a chordal graph is Class 2 if and only if it is neighborhood-overfull. In this paper, we give a characterization of neighborhood-overfull split graphs and we show that the above conjecture is true for some split graphs.
\end{abstract}

Keywords Edge-coloring · Overfull graph · Split graph . Classification problem

\section{Introduction}

An edge-coloring of $G$ is an assignment of one color to each edge of $G$ such that no adjacent edges have the same color. The chromatic index, $\chi^{\prime}(G)$, is the minimum number of colors for which $G$ has an edge-coloring.

S. Morais de Almeida $(\bowtie) \cdot$ C. Picinin de Mello

Institute of Computing, University of Campinas, Campinas, Brazil

e-mail: sheila.almeida@ufms.br

C. Picinin de Mello

e-mail: celia@ic.unicamp.br

S. Morais de Almeida

Campus of Ponta Porã, Federal University of Mato Grosso do Sul, Ponta Porã, Brazil

A. Morgana

Department of Mathematics, University of Rome "La Sapienza",

Roma, Italy

e-mail: morgana@mat.uniromal.it
An easy lower bound for the chromatic index is the maximum vertex degree $\Delta$. A celebrated theorem of Vizing [27] states that, for a simple graph, the chromatic index is at most $\Delta+1$. It was the origin of the Classification Problem that consists of deciding whether a given graph has chromatic index equal to $\Delta$ or $\Delta+1$. Graphs whose chromatic index is equal to $\Delta$ are said to be Class 1; graphs whose chromatic index is equal to $\Delta+1$ are said to be Class 2. Despite the restriction imposed by Vizing, it is NP-complete to determine, in general, if a graph is Class 1 [15]. In 1991, Cai and Ellis [2] proved that this holds also when the problem is restricted to some classes of graphs such as perfect graphs. However, the classification problem is entirely solved for some well-known classes of graphs that include the complete graphs, bipartite graphs [16], complete multipartite graphs [14], and graphs with universal vertices [20]. Nevertheless, the complexity of the classification problem is unknown for several well-studied strongly structured graph classes such as cographs [1], join graphs [17, 24, 25], planar graphs [23], chordal graphs, and several subclasses of chordal graphs such as split graphs [3], indifference graphs, interval graphs, and doubly chordal graphs [9].

By Vizing's theorem, to show that a graph $G$ is Class 1 it is enough to construct an edge-coloring for $G$ with $\Delta(G)$ colors, however, to show that $G$ is Class 2 we must prove that $G$ does not have an edge-coloring with $\Delta(G)$ colors. Considering a simple graph $G$, the inequality $|E(G)|>$ $\Delta(G)\left\lfloor\frac{\mid V(G)\rfloor}{2}\right\rfloor$ is a useful sufficient condition to classify $G$ as a Class 2 graph. In such a way, this condition implies that $G$ has "many edges" and it is called overfull graph. Note that if a graph $G$ is overfull, then $G$ has an odd number of vertices and, since at most $\left\lfloor\frac{|V(G)|}{2}\right\rfloor$ edges of $G$ can be colored with the same color, it is Class 2. Moreover, if a graph $G$ has an overfull subgraph $H$ with $\Delta(H)=\Delta(G)$, it is a subgraph-overfull graph [11]. When the overfull sub- 
graph $H$ is induced by a $\Delta(G)$-vertex $v$ and all its neighbors, denoted by $N[v]$, we say that $G$ is a neighborhood-overfull graph [8]. Overfull, subgraph-overfull, and neighborhoodoverfull graphs are Class 2. Although very rare, there are examples of Class 2 graphs that are neither subgraph-overfull nor neighborhood-overfull. The smallest one is $P^{*}$, the graph obtained from the Petersen graph by removing an arbitrary vertex.

Hilton and Chetwynd [4] conjectured that being Class 2 is equivalent to being subgraph-overfull, when the graph has a maximum degree greater than $\frac{|V(G)|}{3}$. This conjecture is known as the Overfull Conjecture. Every Class 2 graph with maximum degree at least $|V(G)|-3$ is subgraphoverfull [5]; every Class 2 complete multipartite graph is overfull [14]. These classes provide evidence for the Overfull Conjecture. Note that if the Overfull Conjecture is true, the resulting theorem can not be improved, since $\frac{\left|V\left(P^{*}\right)\right|}{3}=$ $\Delta\left(P^{*}\right)$.

A split graph is a graph whose vertex set admits a partition into a clique and a stable set. Split graphs are a well-studied class of graphs for which most combinatorial problems are solved $[6,7,18,19,22]$. It has been proved that every overfull split graph contains a universal vertex and, therefore, is neighborhood-overfull. Moreover, every subgraph-overfull split graph is in fact neighborhoodoverfull [8]. In the same article, the authors have posed the following conjecture for chordal graphs (graphs without induced cycles $C_{n}$ with $n \geq 4$ ), a superclass of split graphs.

\section{Conjecture 1 Every Class 2 chordal graph is neigh- borhood-overfull.}

Note that the validity of this conjecture for chordal graphs and, therefore, for split graphs implies that the edge-coloring problem for the corresponding class is in $P$.

In this work, we present a structural characterization of the neighborhood-overfull split graphs. If Conjecture 1 is true for split graphs, we are presenting a structural characterization of the unique Class 2 split graphs.

The study of the core and the semi-core of a graph gives us some information about the Classification Problem. The core of a graph $G$, denoted by $G_{\Delta}$, is the subgraph of $G$ induced by the $\Delta(G)$-vertices. The core of a graph has been studied, since 1965, when Vizing [28] proved that $G$ is Class 1 if $G_{\Delta}$ has at most two vertices. This result was later generalized by Fournier [10]: if $G_{\Delta}$ is a forest, then $G$ is Class 1 . Thus, the question was what happens when the core of a graph contains a cycle. Hilton and Zhao [12, 13] considered the graphs whose core is the disjoint union of cycles and paths, i.e., $\Delta\left(G_{\Delta}\right)=2$ and they conjectured that every graph with $\Delta\left(G_{\Delta}\right)=2$, different from $P^{*}$, is Class 2 if and only if it is overfull. Tan and Hung [26] proved that this conjecture is true for split graphs. Note that a split graph with $\Delta\left(G_{\Delta}\right)=2$ has 3 vertices with maximum degree.
The semicore of a graph $G$ is the subgraph induced by the core of $G$ and their neighbors. An interesting result says that the chromatic index of a graph is equal to the chromatic index of its semicore [17]. In general, to solve the Classification Problem for the semicore of $G$ is as hard as to solve the Classification Problem for $G$. However, under special conditions of the semicore, a useful tool to solve the Classification Problem for $G$ is to solve it for its semicore. We use this approach to classify some split graphs.

In Sect. 2, we recall some known results that we use in the subsequent sections. In Sect. 3, we give a characterization of neighborhood-overfull split graphs, and in Sect. 4, we show that Conjecture 1 is true for some subclasses of split graphs.

\section{Theoretical framework}

In this paper, $G$ denotes a simple, finite, undirected, and connected graph with vertex set $V(G)$ and edge set $E(G)$. Write $n=|V(G)|$ and $m=|E(G)|$. A subgraph $H$ of a graph $G$ is a graph with $V(H) \subseteq V(G)$ and $E(H) \subseteq E(G)$. For $X \subseteq V(G)$, denote by $G[X]$ the subgraph induced by $X$, that is, $V(G[X])=X$ and $E(G[X])$ consists of those edges of $E(G)$ having both ends in $X$. Let $D \subseteq E(G)$. The subgraph induced by $D$ is the subgraph $H$ with $E(H)=D$ and $V(H)$ is the set of every vertex of $G$ with at least one edge of $D$ incident to it. For any $v$ in $V(G)$, the set of vertices adjacent to $v$ is denoted by $N(v)$ and $N[v]=\{v\} \cup N(v)$. The subgraph induced by $N(v)$ and $N[v]$ are called neighborhood of $v$ and closed neighborhood of $v$, respectively. Two vertices, $u$ and $v$, of a graph $G$ are twin vertices if $N[u]=N[v]$ in $G$. For $X \subseteq V(G), N(X)=\bigcup_{v \in X} N(v)$. The degree of a vertex $v$ is $d_{G}(v)=|N(v)|$. The maximum degree of $G$ is $\Delta(G)=\max \left\{d_{G}(v): v \in V(G)\right\}$. A $\Delta(G)$ vertex is a vertex $v$ with $d_{G}(v)=\Delta(G)$. When there is no ambiguity, we remove the symbol $G$ from the notation.

A clique is a set of pairwise adjacent vertices of a graph. A maximal clique is a clique that is not properly contained in any other clique. A stable set is a set of pairwise nonadjacent vertices. A split graph $G=\{Q, S\}$ is a graph whose vertex set admits a partition $\{Q, S\}$ into a clique $Q$ and a stable set $S$.

In the following, we shall use some known results that we recall for reader's convenience.

Theorem 2 [20] Let $G$ be a graph with $\Delta(G)=|V(G)|$ -1 . Then $G$ is Class 1 if and only if $|E(\bar{G})| \geq \frac{\Delta(G)}{2}$.

Theorem 3 [17] The chromatic index of a graph $G$ is equal to the chromatic index of its semicore.

Theorem 4 [3] Let $G=\{Q, S\}$ be a split graph. If $\Delta(G)$ is odd, then $G$ is Class 1. 
Theorem 5 [8] Let $G=\{Q, S\}$ be a split graph. If $G$ is overfull, then $G$ has a universal vertex. Moreover, $G$ is subgraph-overfull if and only if $G$ is neighborhood-overfull.

Theorem 6 [26] Let $G=\{Q, S\}$ be a split graph. Let $X$ be the set of $\Delta(G)$-vertices. If $|N(A) \cap S| \geq|A|$, for each $A \subseteq X$, then $G$ is Class 1 .

Theorem 7 [26] Let $G=\{Q, S\}$ be a split graph with $\Delta\left(G_{\Delta}\right) \leq 2$. Then $G$ is Class 2 if and only if $G$ is overfull.

\section{A Class 2 split graph}

By Theorem 5, neighborhood-overfull and subgraph-overfull concepts are equivalent when restricts to split graphs. In this section, we give a structural characterization of split graphs that are neighborhood-overfull. As far as we know, these graphs are the unique known Class 2 split graphs.

From now on, we consider a split graph $G=\{Q, S\}$, where $Q$ is a maximal clique and $S$ is a stable set. We shall associate to $G$ a bipartite graph $B$ obtained from $G$ by removing all edges of the subgraph of $G$ induced by $Q$. Let $d(Q)$ be the maximum degree of a vertex of $Q$ in the bipartite graph $B$, i.e., $d(Q)=\max \left\{d_{B}(v): v \in Q\right\}$. Then $\Delta(G)=|Q|-1+d(Q)$.

Lemma 8 Let $G=\{Q, S\}$ be a split graph. If $G$ is a neighborhood-overfull graph, then $|Q|$ and $d(Q)$ must have different parities and $|Q|=(d(Q))^{2}+i$ with $i$ odd, $i \geq 3$.

Proof Let $G=\{Q, S\}$ be a split graph. If $\Delta(G)$ is odd, by Theorem $4, G$ is Class 1 and, therefore, $G$ is not neighborhood-overfull. Hence, $\Delta(G)=|Q|+d(Q)-1$ must be even. This implies that $|Q|$ and $d(Q)$ have different parities.

Assume that $G$ is neighborhood-overfull. If $G$ is a complete graph, it is known that $|Q|$ must be odd with $|Q| \geq 3$ and the lemma follows. Therefore, we consider $S \neq \emptyset$. Since $G$ is neighborhood-overfull, $G$ contains a $\Delta(G)$ vertex $v$ such that $G[N[v]]$ is overfull. Hence, by Theorem $2,|E(\overline{G[N[v]]})| \leq \frac{\Delta(G)}{2}-1$. Since $Q$ is a maximal clique, for each $u \in N[v] \cap S$ there exists at least one vertex $w \in Q$ such that $\{u, w\} \notin E(G)$. Then $\left(\begin{array}{c}d(Q) \\ 2\end{array}\right)+$ $d(Q) \leq|E(\overline{G[N[v]]})| \leq \frac{\Delta(G)}{2}-1$. This implies that $|Q| \geq$ $(d(Q))^{2}+3$. Moreover, the parities of $|Q|$ and $d(Q)$ imply that $|Q|=(d(Q))^{2}+i$ with $i$ odd, $i \geq 3$.

Now we give a characterization of neighborhood-overfull split graphs. It is relevant to note that the next theorem guarantees that every neighborhood-overfull split graph $G$ contains a minimum number of $\Delta(G)$-vertices that have the same neighborhood.
Theorem 9 Let $G=\{Q, S\}$ be a split graph. The graph $G$ is neighborhood-overfull if and only if the following conditions hold:

1. $\Delta(G)$ is even; and

2. there exists a set $X \subseteq Q$ with at least $k=|Q|-\frac{\Delta(G)}{2}+$ $\left(\begin{array}{c}d(Q) \\ 2\end{array}\right)+1 \Delta(G)$-vertices that are twins and, for a $v \in X$, the number of edges of $\overline{G[N[v]]}$ incident to vertices of $Q \backslash X$ is at most $|Q|-k$.

Proof Let $G=\{Q, S\}$ be a split graph. Suppose that $G$ is neighborhood-overfull.

If $G$ is a complete graph, every vertex is a $\Delta(G)$-vertex and all the conditions are trivially true. Therefore, we consider the case $S \neq \emptyset$. Since $G$ is neighborhood-overfull, $G$ contains a $\Delta(G)$-vertex $v$ such that $G[N[v]]$ is overfull. Then condition (1) is true. Moreover, by Theorem 2, $|E(\overline{G[N[v]]})|<\frac{\Delta(G)}{2}$. So, there are at most $\frac{\Delta(G)}{2}-1-$ $\left(\begin{array}{c}d(Q) \\ 2\end{array}\right)$ vertices in $Q$ which are not adjacent to at least one vertex in $N[v] \cap S$. Therefore, $G[N[v]]$ contains at least $k=|Q|-\frac{\Delta(G)}{2}+\left(\begin{array}{c}d(Q) \\ 2\end{array}\right)+1$ vertices of maximum degree. Let $X$ be the set of the vertices of maximum degree in $G[N[v]](|X| \geq k)$. Since $v$ is a $\Delta(G)$-vertex, $\Delta(G)=\Delta(G[N[v]])$. Since $|N[v] \cap S|=d(Q)$, all vertices in $X$ are $\Delta(G)$-vertices and they are twins. Furthermore, $\sum_{w \in Q \backslash X} d \overline{G[N[v]]}(w) \leq \frac{\Delta(G)}{2}-1-\left(\begin{array}{c}d(Q) \\ 2\end{array}\right)=|Q|-k$.

Now suppose that conditions (1) and (2) are true. Let $v$ be one of the $k$ vertices of maximum degree that are twins and consider $G[N[v]]$. By condition (2), we have $|E(\overline{G[N[v]]})| \leq\left(\begin{array}{c}d(Q) \\ 2\end{array}\right)+|Q|-k=\frac{\Delta(G)}{2}-1$. By condition (1), $G$ has even maximum degree. Therefore, $G$ is a neighborhood-overfull graph.

The split graphs described in Theorem 9 are Class 2 . Therefore, if the Conjecture 1 were true, these graphs would be the unique Class 2 split graphs and every split graph $G=\{Q, S\}$ with $\Delta(G)$ even and $|Q|<(d(Q))^{2}+3$ would be Class 1 .

Corollary 10 Let $G=\{Q, S\}$ be a neighborhood-overfull split graph. Then $\Delta(G)>\frac{|V(G)|}{3}$.

Proof Let $G=\{Q, S\}$ be a neighborhood-overfull split graph. By Theorem $9, G$ has a set $X$ of twin $\Delta(G)$-vertices such that $\left|N_{G}(X) \cap S\right|=d(Q)$. Moreover, by Theorem 9, there are at most $\left(\frac{\Delta(G)}{2}-1\right)-\left(\begin{array}{c}d(Q) \\ 2\end{array}\right)$ edges in $\bar{G}$ with an end in $Q \backslash X$ and another one in $N_{G}(X) \cap S$. Note that for each one of these edges could exist an edge in $G$ with an endpoint in $Q \backslash X$ and another one in $S \backslash N(X)$. We call by $E^{\prime}$ this set of edges of $G$. So, there are at most $\left(\frac{\Delta(G)}{2}-1\right)-\left(\begin{array}{c}d(Q) \\ 2\end{array}\right)$ vertices of $S$ that are endpoints of edges of $E^{\prime}$. Note that each vertex of $S$ belongs to $N(X)$ or it is endpoint of an edge of $E^{\prime}$. Note also that the cardinality of $S$ increases if the vertices that are endpoints of edges of $E^{\prime}$ are all distinct. So, the 
maximum cardinality of $S$ is $d(Q)+\left(\frac{\Delta(G)}{2}-1\right)-\left(\begin{array}{c}d(Q) \\ 2\end{array}\right)$, where $d(Q)$ is the number of vertices belonging to $N(X)$ and $\left(\frac{\Delta(G)}{2}-1\right)-\left(\begin{array}{c}d(Q) \\ 2\end{array}\right)$ is the maximum number of vertices belonging to $S \backslash N(X)$ which are endpoints of edges of $E^{\prime}$. Since $|S| \leq d(Q)+\left(\frac{\Delta(G)}{2}-1\right)-\left(\begin{array}{c}d(Q) \\ 2\end{array}\right)$, we have $|V(G)|=|Q|+|S| \leq|Q|+d(Q)-1+\frac{\Delta(G)}{2}-\left(\begin{array}{c}d(Q) \\ 2\end{array}\right)$. Recall that $\Delta(G)=|Q|+d(Q)-1$. So, $V(G) \leq \Delta(G)+$ $\frac{\Delta(G)}{2}-\left(\begin{array}{c}d(Q) \\ 2\end{array}\right)$. Therefore, $\Delta(G) \geq 2\left(\frac{|V(G)|}{3}\right)+\frac{2}{3}\left(\begin{array}{c}d(Q) \\ 2\end{array}\right)>$ $\frac{|V(G)|}{3}$.

The split graphs described in Theorem 9 are Class 2 . Therefore, if the Conjecture 1 were true, these graphs would be the unique Class 2 split graphs and every split graph $G=\{Q, S\}$ with $\Delta(G)$ even and $|Q|<(d(Q))^{2}+3$ would be Class 1 .

\section{Some Class 1 split graphs}

In this section, we use the semi-core of a given split graph $G$ to determine the chromatic index of $G$. In general, finding the chromatic index of the semicore of a graph $G$ may be as difficult as finding the chromatic index of $G$. If $G$ has the hereditary property for induced subgraphs, $G$ and its semicore belongs to the same class. This is the case of split graphs. However, under special conditions this approach may be useful for classifying certain split graphs.

Theorem 11 Let $G=\{Q, S\}$ be a split graph and let $H$ be the semicore of $G$. If $H$ has a universal vertex, then $G$ is Class 2 if and only if $H$ is overfull.

Proof Let $G=\{Q, S\}$ be a split graph and let $H$ be the semicore of $G$. Then $H$ is also a split graph and $\Delta(H)=$ $\Delta(G)$. To calculate the chromatic index of $G$, by Theorem 3, it is sufficient to calculate the chromatic index of its semicore. By the hypothesis, $H$ has a universal vertex. So, by Theorem 2, $H$ is Class 2 if and only if $H$ is overfull. Therefore, $G$ is Class 2 if and only if $H$ is overfull.

By Theorem 11, the Classification Problem is solved for a split graph if its semicore has a universal vertex. Now, we consider split graphs whose semicore has no universal vertex.

Lemma 12 Let $G=\{Q, S\}$ be a split graph. Let $X$ be the set of $\Delta(G)$-vertices. Let $H$ be the semicore of $G$ and suppose that $H$ does not contain universal vertices. If $\Delta(G) \geq$ $|Q|+|X|-2$, then for each $A \subseteq X,|N(A) \cap S| \geq|A|$.

Proof Let $H$ be the semicore of a split graph $G=\{Q, S\}$ and let $X$ be the set of $\Delta(G)$-vertices. Then $H$ is a split graph with partition $\{Q, N(X) \cap S\}$. Since $\Delta(G) \geq|Q|+$
$|X|-2, d(Q) \geq|X|-1$. A $\Delta(G)$-vertex is also a $\Delta(H)$ vertex, then $d(Q) \geq|X|-1$. By hypothesis, $H$ has no universal vertex, so there exist two distinct vertices $v_{i}, v_{j}$ in $X$ such that $N_{H}\left(v_{i}\right) \neq N_{H}\left(v_{j}\right)$. Therefore, $|N(X) \cap S| \geq$ $d(Q)+1$. Since $d(Q)+1 \geq|X|,|N(X) \cap S| \geq|X|$.

Since $d(Q) \geq|X|-1$, if $|A|=|X|,|N(A) \cap S|=$ $|N(X) \cap S| \geq|X|$, and, for each proper subset $A \subset X$ such that $|A| \leq|X|-1,|N(A) \cap S| \geq|X|-1$. Therefore, the lemma follows.

The next theorem shows that the Classification Problem is also solved for split graphs $G$ that have $\Delta(G)$ even and $\Delta(G) \geq|Q|+|X|-2$, where $X$ is the set of $\Delta(G)$-vertices.

Theorem 13 Let $G=\{Q, S\}$ be a split graph. Let $X$ be the set of $\Delta(G)$-vertices. If $\Delta(G) \geq|Q|+|X|-2$, then $G$ is Class 2 if and only if $G$ is neighborhood-overfull.

Proof Let $G=\{Q, S\}$ be a split graph, let $H$ be the semicore of $G$ and let $X$ be the set of $\Delta(G)$-vertices.

If $H$ has a universal vertex, then by Theorem 2, $G$ is Class 2 if and only if $H$ is overfull. If $H$ is overfull, by definition of neighborhood-overfull graph, $G$ is neighborhoodoverfull.

Now consider $H$ without universal vertices. By the hypothesis, $\Delta(G) \geq|Q|+|X|-2$. Then, by Lemma 12 and Theorem $6, H$ is Class 1 . By Theorem 3, $G$ is Class 1 . Therefore, $G$ is Class 2 if and only if $G$ is neighborhoodoverfull.

Now, we consider a split graph $G=\{Q, S\}$ with $|X| \geq 4$ vertices of maximum degree and an even $\Delta(G)=|Q|+$ $|X|-3$. Note that $\Delta\left(G_{\Delta}\right) \geq 3$. (Recall that Tan and Hung [26] proved that when $\Delta\left(G_{\Delta}\right)=2$, a split graph $G$ is Class 2 if and only if $G$ is overfull.) First, we show that, when $\Delta\left(G_{\Delta}\right) \geq 3$ and $\Delta(G)=|Q|+|X|-3, G$ is not neighborhood-overfull. After we show that, if $|X|$ is odd, then $G$ is Class 1 .

Lemma 14 Let $G=\{Q, S\}$ be a split graph with even maximum degree. Let $X$ be the set of $\Delta(G)$-vertices with $|X| \geq 4$. If $\Delta(G)=|Q|+|X|-3$, then $G$ is not neighborhoodoverfull.

Proof Let $G=\{Q, S\}$ be a split graph with $|X| \geq 4$ vertices of maximum degree and let $\Delta(G)=|Q|+|X|-3$ be an even number. Then $d(Q)=|X|-2$. If $G$ is neighborhoodoverfull, then by Lemma 8 , the condition $|Q| \geq(d(Q))^{2}+3$ implies that $|Q| \geq(|X|-2)^{2}+3$ and, by Theorem 9 , the condition $|Q|-\frac{\Delta(G)}{2}+\left(\begin{array}{c}d(Q) \\ 2\end{array}\right)+1 \leq|X|$ implies $|Q| \leq$ $-|X|^{2}+8|X|-11$. These inequalities imply that there exists a neighborhood-overfull split graph if and only if $|X|=3$. Therefore, $G$ is not neighborhood-overfull. 
Lemma 15 Let $G=\{Q, S\}$ be a split graph with even maximum degree. Let $X$ be the set of $\Delta(G)$-vertices with $|X|>4$. If $\Delta(G)=|Q|+|X|-3$ and $|X|$ is odd, then $G$ is Class 1 .

Proof Let $G=\{Q, S\}$ be a split graph with even maximum degree. Let $X$ be the set of $\Delta(G)$-vertices with odd $|X|>4$. Since $\Delta(G)=|Q|+|X|-3, d(Q)=|X|-2$. By Lemma $14, G$ is not neighborhood-overfull and we shall prove that $G$ is Class 1 .

To calculate the chromatic index of $G$, by Lemma 3, it is sufficient to calculate the chromatic index of its semicore. Let $X=\left\{v_{0}, \ldots, v_{|X|-1}\right\}$ be the set of $\Delta(G)$-vertices. Since $Q$ is maximal, $X \subseteq Q$. Consider the semicore $H$ of $G$. By definition of semi-core, $H=\left\{Q, S_{H}\right\}$ is a split graph with partition $Q$ and $S_{H}=N(X) \cap S$, and has $\Delta(H)=\Delta(G)=$ $\Delta$. Since $G$ is not neighborhood-overfull, for each $\Delta$-vertex $v, G[N[v]]$ is not overfull. Then $H$ is not neighborhoodoverfull, because $G[N[v]]=H[N[v]]$. If $H$ has a universal vertex, then by Theorem $11, H$ is Class 1 .

Now, we assume that $H$ has no universal vertex, so there is a $\Delta$-vertex $v$ such that $N[v] \neq N[w], w \in X$. Thus, $\left|S_{H}\right| \geq d(Q)+1=|X|-1$. If $|N(A) \cap S| \geq|A|$ for every $A \subseteq X$, then, by Theorem $6, H$ is Class 1 and the lemma follows. It remains to consider the case when there is a subset $A$ of $X$ such that $|N(A) \cap S|<|A|$. In this case, we shall give an edge-coloring of $H$ with $\Delta$ colors by constructing from $H$ a suitable graph with odd maximum degree equal to $\Delta-1$.

Since $d(Q)=|X|-2$, the condition $|N(A) \cap S|<|A|$ may occur when $|A|=|X|-1$ and $|N(A) \cap S|=|X|-2$ or $|A|=|X|$ and $|N(A) \cap S|=|X|-1$. In both cases, there are at least two $\Delta$-vertices that are twins. We call them $y$ and $z$. Note that $\left|N(y) \cap S_{H}\right|=\left|N(z) \cap S_{H}\right|=d(Q)$.

Let $u$ be a vertex with minimum degree belonging to $N(y) \cap S_{H}$. Now consider the edges $y u$ and $z u$. Construct a set $M^{\prime}=M \cup\{y u, z u\}$, where $M$ is a matching with $|X|-2$ edges such that each edge of $M$ is incident to a $\Delta$-vertex $v$, distinct from $y$ and $z$, and no edge in $M$ is incident to $u$. Note that both $M \cup\{y u\}$ and $M \cup\{z u\}$ are matchings.

Now, we construct from $H$ a split graph $L=\left\{Q, S_{L}\right\}$. First, make a copy $L$ from $H \backslash M^{\prime}$. Then, for each $v \in Q$ with $d_{L}(v)<\Delta-1$, add new vertices of degree 1 in $S_{L}$ adjacent to $v$ until $d_{L}(v)=\Delta-1$. Note that $\Delta(L)=\Delta-1$, $S_{L}$ has more vertices than $S_{H}$, and every vertex of $Q$ is a $\Delta(L)$-vertex.

We use the algorithm of $\mathrm{Chen}, \mathrm{Fu}$, and $\mathrm{Ko}$, with a $(\Delta-$ 1) $\times(\Delta-1)$ Latin square $L S=\left(m_{i j}\right)$ defined by $m_{i j}=i+j$ $(\bmod \Delta-1), 0 \leq i, j \leq \Delta-2$, to give an edge-coloring $\pi^{\prime}$ for $L$ with $\Delta-1$ colors.

In order to give this edge-coloring for $L$, they use a special ordering of the vertices of $L[Q]$ constructed as follows. Consider an ordering $\left(u_{0}, u_{1}, \ldots, u_{\left|S_{L}\right|-1}\right)$ of the vertices of $S_{L}$ such that $N_{L}(y) \cap S_{L}$ are the first ones and $u$ is the last one. Let $U=\left(u_{0}, u_{1}, \ldots, u_{h}\right)$, where $h$ is the minimum number with $|N(U)| \geq|Q| / 2$. Let $B$ be the bipartite subgraph of $L$ with partition $\{Q, U\}$ and edges with an end in $Q$ and another one in $U$. A CFK-ordering is an ordering of the vertices of $Q$ such that $d_{B}\left(v_{i}\right) \geq d_{B}\left(v_{i+1}\right)$ for each $i=0, \ldots,|Q|-1$. Moreover, if $d_{B}\left(v_{i}\right)=d_{B}\left(v_{j}\right)$, $v_{i} u_{h} \in E(L)$, and $v_{j} u_{h} \notin E(L)$, then $i>j$.

We show that there exists a CFK-ordering of the vertices of $Q$ such that $y$ and $z$ are the first ones in the ordering. If $\left|N_{B}(y)\right|<|U|=h+1$, then $d_{B}(y)=d_{B}(z)=d_{B}(Q)$, where $d_{B}(Q)$ is the maximum degree of vertex of $Q$ in the subgraph $B$. Hence, $d_{B}(v) \leq d_{B}(y)$ for each $v \in Q$. Moreover, if $\left|N_{B}(y)\right|<h+1$, then $y u_{h} \notin E(L)$ and $z u_{h} \notin E(L)$. Therefore, $y$ and $z$ can be chosen as the first vertices in a CFK-ordering. If $\left|N_{B}(y)\right| \geq h+1$, then $d_{B}(y)=d_{B}(z)=$ $|U|$ and again $d_{B}(v) \leq d_{B}(y)$ for each $v \in Q$. Moreover, since $d_{B}(y)=d_{B}(z)=|U|, y u_{h} \in E(L)$ and $z u_{h} \in E(L)$ and if $d_{B}(v)=d_{B}(y)(v \neq y$ and $v \neq z)$, then $v u_{h} \in E(L)$. Again, $y$ and $z$ can be chosen as the first vertices in a CFKordering.

Now, consider the vertices of $Q$ sorted in a CFK-ordering where $y$ and $z$ are the first. Using the algorithm of $\mathrm{Chen}, \mathrm{Fu}$, and Ko [3], the colors of the first $|Q|$ lines and columns of the Latin square $L S$ are assigned to the edges of $L[Q]$ so that an edge $v_{i} v_{j}$ in $E(L[Q])$ is colored by the color $m_{i j}$. The remaining column entries and the entries of the main diagonal of $L S$ are used to color the edges with an end vertex in $Q$ and another in $S_{L}$ as follows. The edges $v_{i} u_{h}$ are colored with color $m_{i i}, 0 \leq i<|Q|$. The remaining colors of the main diagonal (not used when the edge $v_{i} u_{h}$ does not exist in $L$ ) and the colors of the first $|Q|$ lines and last $\Delta-|Q|$ columns of $L S$ are used to color the edges $v_{i} u$, when $u \neq u_{h}$.

By definition of Latin square $L S$ and considering the coloring applied to $L[Q]$, we can observe that each edge $v_{i} v_{j} \in Q$ such that $i+j=|Q|-1(\bmod \Delta-1)$ is colored $|Q|-1$. Since $|X|$ is odd, $\Delta$ is even, and $\Delta=|Q|+|X|-3$, $|Q|$ is even. Since $|Q|$ is even, each edge incident to a vertex of $S_{L}$ has a color distinct from $|Q|-1$.

Now, we assign colors to each edge $v w$ of $H$. For this, consider the vertices of $H$ in the same order they are in $L$. We must distinguish two cases depending on whether $v_{|Q|-1}$ is a $\Delta$-vertex or not. Note that in the first case there exists an edge $v_{|Q|-1} u^{\prime} \in M$. Since $M \cup\left\{v_{0} u\right\}$ is a matching, $u \neq u^{\prime}$.

The colors are assigned by $\pi$, for each $v w \in E(G)$, as follows.

- If $v_{|Q|-1}$ is not a $\Delta$-vertex, then $\pi(v w)$ is

$$
\begin{cases}\pi^{\prime}(v w), & \text { if } v w \notin M^{\prime} \text { and } v w \neq v_{0} v_{|Q|-1} \\ \Delta-1, & \text { if } v w \in M^{\prime} \text { and } v w \neq v_{0} u \\ \Delta-1, & \text { if } v w=v_{0} v_{|Q|-1} \\ |Q|-1, & \text { if } v w=v_{0} u\end{cases}
$$


- If $v_{|Q|-1}$ is a $\Delta$-vertex, then $\pi(v w)$ is

$$
\begin{cases}\pi^{\prime}(v w), & \text { if } v w \notin M^{\prime} \text { and } v w \neq v_{0} v_{|Q|-1} ; \\ \Delta-1, & \text { if } v w \in M^{\prime} \text { and } \\ & v w \neq v_{0} u \text { and } v w \neq v_{|Q|-1} u^{\prime} ; \\ \Delta-1, & \text { if } v w=v_{0} v_{|Q|-1} ; \\ |Q|-1, & \text { if } v w=v_{0} u \text { and } v w=v_{|Q|-1} u^{\prime} .\end{cases}
$$

If $v_{|Q|-1}$ is not a $\Delta$-vertex, then the set of edges $\left(M^{\prime} \backslash\right.$ $\left.\left\{v_{0} u\right\}\right) \cup\left\{v_{0} v_{|Q|-1}\right\}$ is a matching, because $M \cup\left\{v_{1} u\right\}$ is a matching. Otherwise, since $X \subseteq Q$ and $\Delta=|Q|+|X|-3$ is an even integer, $|Q|>|X|>4$ and $v_{|Q|-1} \neq v_{1}$, the set of edges $\left(M^{\prime} \backslash\left(\left\{v_{0} u\right\} \cup\left\{v_{|Q|-1} u^{\prime}\right\}\right)\right) \cup\left\{v_{0} v_{|Q|-1}\right\}$ is a matching. In both cases, the edges of these sets can be colored with the new color $\Delta-1$.

Since $|Q|$ is even, no edge incident to a vertex of $S_{H}$ is colored $|Q|-1$. Since $u \neq u^{\prime}$, we can color the edge $v_{0} u$ and the edge $v_{|Q|-1} u^{\prime}$, if it exists, with color $|Q|-1$.

Therefore, $H$ has an edge-coloring with $\Delta$ colors and, by Lemma 3, $G$ is Class 1 .

Theorem 16 Let $G=\{Q, S\}$ be a split graph with even maximum degree. Let $X$ be the set of $\Delta(G)$-vertices. If $\Delta(G) \geq|Q|+|X|-3$ and $|X|$ is odd, then $G$ is Class 2 if and only if $G$ is neighborhood-overfull.

Proof If $\Delta(G) \geq|Q|+|X|-2$, then, by Theorem 13, $G$ is Class 2 if and only if $G$ is neighborhood-overfull.

Let $\Delta(G)=|Q|+|X|-3$. Since $\Delta(G)=|Q|+d(Q)$ $-1, d(Q)=|X|-2$. Since, $d(Q) \geq 0$ and, by hypothesis, $|X|$ is odd, $|X|>2$. If $|X|=3$, then $\Delta\left(G_{\Delta}\right)=2$ and, by Theorem 7, $G$ is Class 2 if and only if $G$ is neighborhoodoverfull. Finally, if $|X|>4$ and $|X|$ is odd, the theorem follows, by Lemma 15.

\section{Conclusions}

The split graphs described in Theorem 9 are Class 2. If the Conjecture 1 were true, then the graphs described in Theorem 9 would be the unique Class 2 split graphs.

By Corollary 10, every neighborhood-overfull split graph has $\Delta(G) \geq \frac{|V(G)|}{3}$. So, if Conjecture Overfull were true for split graphs, it provides a strong evidence that Conjecture 1 were true for these graphs.

Theorem 16 gives another evidence that Conjecture 1 is true, providing a polynomial algorithm for edge-coloring with $\Delta$ colors the graphs of a new set of non neighborhoodoverfull split graphs. It is known that if $G$ is a graph with $\Delta(G) \geq|V(G)|-3$, then $G$ is Class 1 if and only if $G$ is not subgraph-overfull $[5,20,21]$. For a split graph $G$ with $\Delta(G)$ even, the condition on $\Delta(G)$, given by Theorem 16 , can be smaller than $|V(G)|-3$.
Acknowledgements We are grateful to anonymous referees for their careful reading and valuable suggestions, which helped improve an earlier version of this note. This research was partially supported by CAPES and CNPq (140709/2008-8, 482521/2007-4, and 473867/2010-9). The first author was also partially supported by PROPP/UFMS

\section{References}

1. Barbosa MM, de Mello CP, Meidanis J (1998) Local conditions for edge-colouring of cographs. Congr Numer 133:45-55

2. Cai L, Ellis JL (1991) NP-completeness of edge-colouring some restricted graphs. Discrete Appl Math 30:15-27

3. Chen B-L, Fu H-L, Ko MT (1995) Total chromatic number and chromatic index of split graphs. J Comb Math Comb Comput 17:137-146

4. Chetwynd AG, Hilton AJW (1986) Star multigraphs with three vertices of maximum degree. Math Proc Camb Philos Soc 100:300-317

5. Chetwynd AG, Hilton AJW (1989) The edge-chromatic class of graphs with even maximum degree at least $|V|-3$. Ann Discrete Math 41:91-110

6. Coneil DG, Perl Y (1984) Clustering and domination in perfect graphs. Discrete Appl Math 9(1):27-39

7. Cozzens MB, Halsey MD (1991) The relationship between the threshold dimension of split graphs and various dimensional parameters. Discrete Appl Math 30(2-3):125-135

8. Figueiredo CMH, Meidanis J, de Mello CP (2000) Local conditions for edge-coloring. J Comb Math Comb Comput 32:79-91

9. Figueiredo CMH, Meidanis J, de Mello CP (1999) Totalchromatic number and chromatic index of dually chordal graphs. Inf Process Lett 70:147-152

10. Fournier JC (1973) Coloration des arêtes d'un graphe. Cah Cent étud Rech Opér 15:311-314

11. Hilton AJW (1989) Two conjectures on edge-coloring. Discrete Math 74:61-64

12. Hilton AJW, Zhao C (1992) The chromatic index of a graph whose core has maximum degree two. Discrete Math 101:135-147

13. Hilton AJW, Zhao C (1996) On the edge-colouring of graphs whose core has maximum degree two. J Comb Math Comb Comput 21:97-108

14. Hoffman DG, Rodger CA (1992) The chromatic index of complete multipartite graphs. J Graph Theory 16:159-163

15. Holyer I (1981) The NP-completeness of edge-coloring. SIAM J Comput 10:718-720

16. König D (1916) Über Graphen und ihre Anwendung auf Determinantentheorie und Mengenlehre. Math Ann 77:453-465

17. Machado RCS, Figueiredo CMH (2010) Decompositions for edge-coloring join graphs and cobipartite graphs. Discrete Appl Math 158(12):1336-1342

18. Makino K, Uno Y, Ibaraki T (2006) Minimum edge ranking spanning trees of split graphs. Discrete Appl Math 154(16):2373-2386

19. Ortiz C, Maculan N, Szwarcfiter JL (1998) Characterizing and edge-coloring split-indifference graphs. Discrete Appl Math 82:209-217

20. Plantholt MJ (1981) The chromatic index of graphs with a spanning star. J Graph Theory 5:45-53

21. Plantholt MJ (1983) The chromatic index of graphs with large maximum degree. Discrete Math 47:91-96

22. Royle GF (2000) Counting set covers and split graphs. J Integer Seq $3: 1-5$

23. Sanders DP, Zhao Y (2001) Planar graphs of maximum degree seven are Class I. J Comb Theory, Ser B 83(2):201-212

24. De Simone C, de Mello CP (2006) Edge-colouring of join graphs. Theor Comput Sci 355(3):364-370 
25. De Simone C, Galluccio A (2007) Edge-colouring of regular graphs of large degree. Theor Comput Sci 389(1-2):91-99

26. Tan ND, Hung LX (2006) On colorings of split graphs. Acta Math Vietnam 31(3):195-204
27. Vizing VG (1964) On an estimate of the chromatic class of a $p$ graph. Diskretn Anal 3:25-30

28. Vizing VG (1965) Critical graphs with a given chromatic class. Metody Diskretn Anal 5:9-17 (in Russian) 